\title{
National Lung Cancer Screening Utilization Trends in the Veterans Health Administration
}

\author{
Jennifer A. Lewis (D), MD, MS, MPH, , ,2,3,* Lauren R. Samuels (D), PhD, ${ }^{1,4}$ Jason Denton, ${ }^{1,5,6}$ \\ Gretchen C. Edwards (D), MD, MPH, ${ }^{1,7}$ Michael E. Matheny (D), MD, MS, MPH, ${ }^{1,5,6}$ Amelia Maiga, MD, MPH, ${ }^{7}$ \\ Christopher G. Slatore, MD, MSc, ${ }^{8}$ Eric Grogan, MD, MPH, ${ }^{9}$ Jane Kim (D) MD, MPH, ${ }^{10}$ Robert H. Sherrier, MD, ${ }^{11}$ \\ Robert S. Dittus, MD, MPH, ${ }^{1,6}$ Pierre P. Massion (D), MD, ${ }^{3,12,13}$ Laura Keohane (D) PhD, ${ }^{14}$ Sayeh Nikpay, PhD, ${ }^{14}$ \\ Christianne L. Roumie (D), MD, MPH ${ }^{1,6}$
}

\begin{abstract}
${ }^{1}$ Veterans Affairs Tennessee Valley Healthcare System, Geriatric Research, Education and Clinical Center (GRECC), Nashville, TN, USA, ${ }^{2}$ Division of Hematology/ Oncology, Department of Medicine, Vanderbilt University Medical Center, Nashville, TN, USA, ${ }^{3}$ Vanderbilt Ingram Cancer Center, Nashville, TN, USA, ${ }^{4}$ Department of Biostatistics, Vanderbilt University School of Medicine, Nashville, TN, USA, ${ }^{5}$ Department of Biomedical Informatics, Vanderbilt University Medical Center, Nashville, TN, USA, ${ }^{6}$ Division of General Internal Medicine and Public Health, Vanderbilt University Medical Center,Nashville, TN, USA, ${ }^{7}$ Department of General Surgery, Vanderbilt University Medical Center, Nashville, TN, USA, ${ }^{8}$ Veterans Affairs Portland Health Care System, Center to Improve Veteran Involvement in Care, Pulmonary \& Critical Care Medicine, Portland, Oregon, ${ }^{9}$ Department of Thoracic Surgery, Vanderbilt University Medical Center, Nashville, TN, USA, ${ }^{10}$ Veterans Health Administration, National Center for Health Promotion and Disease Prevention, Durham, NC, USA, ${ }^{11}$ Durham VA Health Care System, Radiology, Durham, NC, USA, ${ }^{12}$ Division of Allergy, Pulmonary, and Critical Care Medicine, Vanderbilt University Medical Center, Nashville, TN, USA, ${ }^{13}$ Department of Medicine, VA Tennessee Valley Healthcare System, Nashville, TN, USA and ${ }^{14}$ Department of Health Policy, Vanderbilt University School of Medicine, Nashville, TN, USA

${ }^{*}$ Correspondence to: Jennifer A. Lewis, MD, MS, MPH, Vanderbilt University Medical Center, 2220 Pierce Avenue, 777 Preston Research Building, Nashville, TN 37232-6307, USA (e-mail: jennifer.a.lewis@vumc.org).
\end{abstract}

\begin{abstract}
Background: Many Veterans are high risk for lung cancer. Low-dose computed tomography (LDCT) is an effective strategy for lung cancer early detection in a high-risk population. Our objective was to describe and compare annual and geographic utilization trends for LDCT screening in the Veteran's Health Administration (VHA). Methods: A national retrospective cohort of screened Veterans from January 1, 2011 to May 31, 2018 was used to calculate annual and regional rates of initial LDCT utilization per 1000 eligible Veterans. We identified Veterans with a first LDCT exam using common procedure terminology codes G0297 or 71250 and described as "lung cancer screening," "screening," or "LCS." The number of screen-eligible Veterans per year was calculated as unique Veterans aged 55 to 80 years seen at a Veterans Affairs medical center (VAMC) in that year, multiplied by 32\% (estimated proportion with eligible smoking history). We present $95 \%$ confidence intervals (CI) for rates. Results: Screened Veterans had a mean age of 66.1 years (standard deviation [SD] = 5.6); 95.5\% male; 77.4\% Caucasian. There were 119300 LDCT exams, of which 80819 (67.7\%) were initial. Nationally, initial screens increased from 0 (95\% CI $=0.00$ to 0.00$)$ in 2011 to $29.6(95 \% \mathrm{CI}=29.26$ to 29.88$)$ scans per 1000 eligible Veterans in $2018\left(P_{\text {trend }}<\right.$ .001). Initial screens increased over time within all geographic regions, most prominently in northeastern and Florida VAMCs. Conclusion: VHA LDCT utilization increased from 2011 to 2018. However, overall utilization remained low. Future interventions are needed to increase lung cancer screening utilization among eligible Veterans.
\end{abstract}

Lung cancer is the leading cause of cancer deaths in the United States (1). The majority (74\%) of cases are diagnosed at advanced stages when few curative treatments are available (1). Early detection with low-dose computed tomography (LDCT) is an effective strategy that detects lung cancer at earlier stages, increasing the chance for cure.

In 2011, the National Lung Screening Trial (NLST) demonstrated a $20 \%$ relative reduction in lung cancer-specific mortality in high-risk individuals (age 55-74, current or former smokers who have quit for less than 15 years, and with at least a 30 packyear history of smoking) screened with annual LDCT compared to chest radiography (2). Despite the NLST results, national estimates suggest that LDCT screening rates are $2 \%-4 \%$ of the eligible population in the United States $(3,4)$. Veterans more frequently use tobacco than civilian counterparts $(5,6)$. The Veterans Health Administration (VHA) follows the US Preventive Services Task 
Force (USPSTF) guidelines, which recommend lung cancer screening for individuals aged 55 to 80 years who are current or former cigarette smokers who quit within the past 15 years and who have at least a 30 pack-year history of smoking (7). The VHA implemented lung cancer screening from 2013 to 2015 at 8 Veterans Affairs medical centers (VAMCs) as part of the Lung Cancer Screening Demonstration Project (8). During this time period, participating VAMCs screened approximately 2100 Veterans and estimated that approximately 900000 Veterans are eligible for screening (9).

Our goal was to examine national and regional trends in annual rates of LDCT utilization for initial lung cancer screening in eligible Veterans during the years following the NLST and the demonstration project.

\section{Methods}

\section{Data Source}

The Observational Medical Outcomes Partnership (OMOP) dataset contains information from Veterans' electronic health records (EHR) and claims data in the national VHA. VA Informatics and Computing Infrastructure (VINCI) hosts a data warehouse of demographic, diagnostic, and procedure information used to identify unique patients and their inpatient and outpatient encounters.

\section{Study Population}

To identify all Veterans age 55 to 80 years who underwent LDCT for lung cancer screening between January 1, 2011 and May 31, 2018 in the VHA, we first identified lung cancer screening exams by selecting records that had current procedural terminology (CPT) codes G0297 or 71250 and that contained a description phrase of "screening," "lung cancer screening," or "LCS" (Supplementary Table 1, available online). Chart review of a random sample determined the positive predictive value (PPV) of the screening LDCT algorithm to be $92 \%$ (95\% confidence intervals $[\mathrm{CI}]=0.81$ to 0.97$)$. We excluded exams missing patient identification numbers or date of birth, performed on Veterans under 55 years old or over 80 years old, or associated with a diagnosis code for lung cancer prior to screening. Because we were interested in unique LDCT screening exams, we excluded subsequent exams.

To estimate the number of Veterans potentially eligible for lung cancer screening based on the USPSTF recommendations in a given year from 2011 to 2018, we obtained the total number of unique Veterans seen annually between the ages of 55 and 80 at each VAMC and nationally.

At each level (VAMC and national), we estimated the number of Veterans meeting the USPSTF smoking history criteria as 32\% of these totals based on previous estimates (9). Year of LDCT and VAMC were the exposure variables.

\section{Characteristics of Screened Patients and Screening Facilities}

Captured patient and facility characteristics included age at initial screening, sex, race or ethnicity, year of examination, Veterans Integrated Service Network (VISN), US Census region, and VAMC complexity score. VAMC complexity score consists of five levels: $1 \mathrm{a}, 1 \mathrm{~b}, 1 \mathrm{c}, 2$, and 3 , where $1 \mathrm{a}$ is the most complex and 3 is the least complex. This ranking system takes into consideration the volume, patient cases, number and type of clinical services, presence and size of residency programs, and research (10). We determined the presence of documented tobacco history and two comorbidities, chronic obstructive pulmonary disease (COPD) and coronary artery disease (CAD), given the prevalence of these diseases in heavy smokers up to 730 days before the LDCT examination date using International Classification of Diseases, Ninth Revision, Clinical Modification (ICD-9$\mathrm{CM})$, CPT, Systematized Nomenclature of Medicine, and Logical Observation Identifiers Names and Codes coding systems (Supplementary Table 3, available online). We also determined the ordering provider's type and specialty.

\section{Statistical Analysis}

Patient, ordering provider, and screening facility characteristics were described using means, standard deviations, and frequencies. We estimated annual rates of initial LDCT per 1000 eligible Veterans at the VAMC and national levels as the total number of initial LDCT exams divided by the estimated number of eligible Veterans in each year at each level. For 2018, because we had screening data for January through May, we calculated projected annual rates based on the January to May data. Although our rates represent the entire population of interest, they still contain some uncertainty due to our screening-classification and eligibility-determination procedures. To acknowledge this uncertainty, we present rates with 95\% CI (Wilson method) $(11,12)$.

To examine changes in annual utilization rates at the national level, we used a chi-squared test for trend in proportions with a pre-specified cutoff for statistical significance of .05. To examine geographic variability, we mapped average annual VAMC-level utilization rates during two periods, 2011-2014 and 2015-2018. To obtain average annual rates during these periods, we first calculated the relevant annual rates, and then took the weighted average of those rates, giving the 2018 values a weight of $5 / 12$ and the values from all other years a weight of 1 . All statistical analysis and mapping was performed using $R$ version 3.6.1 and Stata version 15.1, with zip code data from www.geonames.org.

VA Tennessee Valley Healthcare System's Institutional Review Board approved the study prior to data collection.

\section{Sensitivity Analyses}

We tested the robustness of our definition of a lung cancer screening exam by expanding the definition to include exams with CPT codes G0297 or 71250 and that contained a description phrase of "screening," "lung cancer screening," "LCS," "lowdose," "LDCT," or "VCAR." Volume computed algorithm (VCAR) is a radiology software used to analyze screening LDCTs for three-dimensional volumetric assessment as a reliable approach for non-calcified lung nodules, and exams with this descriptor were possibly lung cancer screening examinations.

We examined initial screening exams just below and above the Medicare eligibility start age of 65 to assess whether the number of VHA screenings declined at the onset of Medicare eligibility. Finally, we stratified our eligible cohort by age ( $<65$ and $\geq 65$ years) to address changes in the age structure of the cohort. 


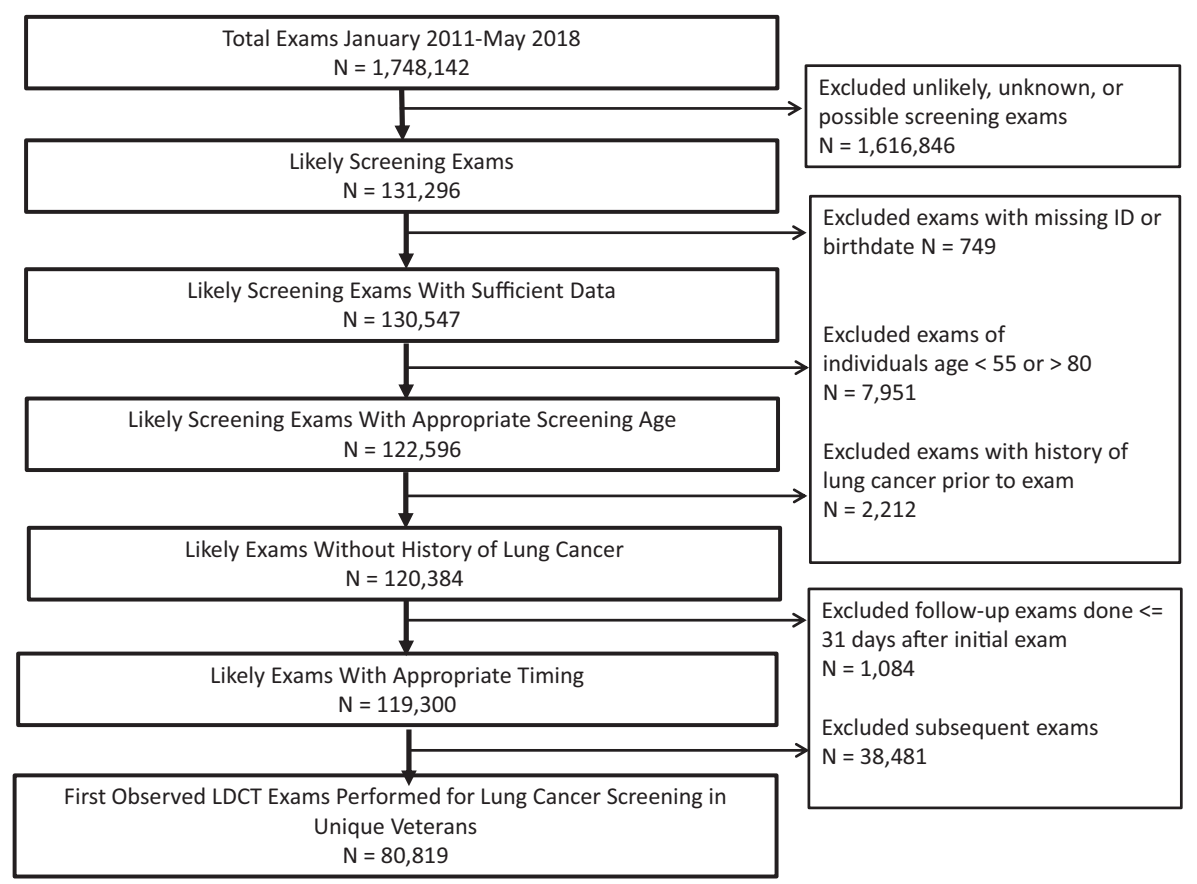

Figure 1. Retrospective cohort flow chart. Time period: January 1, 2011 to May 31, 2018. LDCT = low-dose computed tomography.

\section{Results}

\section{Study Cohort Characteristics at Initial Screening}

We identified 1748142 CT exams performed between 2011 and 2018. We excluded 1616846 exams because the exam was not identified as a screening exam; missing identifier or birthdate $(\mathrm{n}=749)$; age less than 55 or over $80(\mathrm{n}=7951)$; and previous history of lung cancer $(n=2212)$. This resulted in 119300 exams identified as screening; additionally, we excluded subsequent screenings ( $\mathrm{n}=38$ 481). The final cohort included 80819 (67.7\%) initial exams (Figure 1). Of the initial scans, 59138 (73.2\%) were associated with the CPT code G0297, specific for LDCT for lung cancer screening.

At initial screening, Veterans had a mean age of 66.1 years $(\mathrm{SD}=5.6)$. Most screened Veterans were male (95.5\%), Caucasian $(77.4 \%)$, and with documented tobacco use or received medication to assist in quitting in the past 2 years (65.9\%). COPD (36.0\%) and CAD (23.8\%) were prevalent comorbidities (Table 1).

\section{Characteristics of Ordering Providers at Initial Screen and VA Medical Centers}

There were 6759 unique providers who ordered initial LDCT screenings. Most initial LDCT screening exams were ordered by physicians $(77.7 \%)$ and providers within primary care/internal medicine $(55.8 \%)$ or family medicine (15.5\%) (Table 2). There were 75 unique VAMCs that performed initial LDCT screening exams. Of the total number of VAMCs in each region, a higher proportion of northeastern VAMCs (16 out of 23 VAMCs [69.6\%]) performed screenings compared to other regions (31 out of 48 VAMCs [64.6\%] in the south, 16 out of 32 VAMCs [50.0\%] in the west, and 12 out of 27 VAMCs [44.4\%] in the midwest) (Table 3).
Table 1. Veteran characteristics at initial lung cancer screening

\begin{tabular}{lc}
\hline Veteran characteristics & No. of Veterans n (\%) \\
\hline Total & $80819(100)$ \\
Age, mean (SD) & $66.1(5.6)$ \\
Male & $77190(95.5)$ \\
Ethnicity & \\
$\quad$ Not Hispanic or Latino & $73287(90.7)$ \\
Hispanic or Latino & $4636(5.7)$ \\
Missing & $2896(3.6)$ \\
Race & \\
White & $62570(77.4)$ \\
Black or African American & $13354(16.5)$ \\
American Indian or Alaska Native & $534(0.7)$ \\
Asian & $255(0.3)$ \\
Native Hawaiian or other Pacific Islander & $505(0.6)$ \\
$\quad$ Missing & $3601(4.5)$ \\
Documented tobacco use & a \\
Chronic obstructive pulmonary disease & $53293(65.9)$ \\
Coronary artery disease & $29122(36.0)$ \\
\hline
\end{tabular}

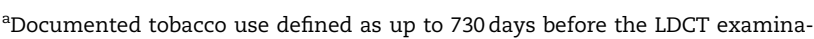
tion date using International Classification of Diseases, Ninth Revision, Clinical Modification (ICD-9-CM), Common Procedural Terminology, Systematized Nomenclature of Medicine, and Logical Observation Identifiers Names and Codes coding systems (see Supplementary Table 3, available online for details). $\mathrm{SD}=$ standard deviation

\section{Lung Cancer Screening Temporal and Geographic Trends}

An estimated 1133183 Veterans in 2011 were eligible for lung cancer screening, which increased to 1171505 in 2018 (Figure 2). At the national level, LDCT screening increased slowly between 2011 and 2014, and more rapidly between 2014 and 2018. In 2011, there were 0 LDCT screenings per 1000 eligible Veterans ( $95 \% \mathrm{CI}=0.00$ to 0.00 ). In 2012 , screening increased to 
Table 2. Ordering provider characteristics at initial lung cancer screening

\begin{tabular}{lcc}
\hline Ordering provider characteristics & No. of unique providers (\%) & No. of initial screenings (\%) \\
\hline Total & $6759(100.0)$ & $80819(100.0)$ \\
Provider type & & \\
Allopathic and osteopathic physician & $5610(83.0)$ & $62787(77.7)$ \\
Advanced practice provider & $924(13.7)$ & $14427(17.9)$ \\
Nursing & $23(0.3)$ & $2779(3.4)$ \\
Other & $19(0.3)$ & $40(0.0)$ \\
Multiple provider types & $4(0.1)$ & $10(0.0)$ \\
Missing & $179(2.6)$ & $776(1.0)$ \\
Ordering provider specialty & & $45097(55.8)$ \\
Primary care/internal medicine & $2198(32.5)$ & $12515(15.5)$ \\
Family medicine & $556(8.2)$ & $779(1.0)$ \\
Geriatrics & $68(1.0)$ & $4242(5.2)$ \\
Allergy, pulmonology, critical care medicine & $203(3.0)$ & $196(0.2)$ \\
Hematology/oncology & $72(1.1)$ & $551(0.7)$ \\
Other internal medicine subspecialist & $179(2.6)$ & $24(0.0)$ \\
Women's health/gynecology & $8(0.1)$ & $8239(10.2)$ \\
Other specialty & $595(8.8)$ & $8329(10.3)$ \\
Physician-in-training & $2696(39.9)$ & $70(0.1)$ \\
Multiple specialties & $4(0.1)$ & $777(1.0)$ \\
Missing & $180(2.7)$ &
\end{tabular}

${ }^{a}$ Other provider includes behavioral health and social services, dental, pharmacy, podiatry and surgery, technicians, and other providers.

0.14 scans (160 exams per 1142508 Veterans; $95 \% \mathrm{CI}=0.12$ to 0.16); 0.58 scans in 2013 (667 exams per 1148060 Veterans; 95\% $\mathrm{CI}=0.54$ to 0.63 ); 4.95 scans in 2014 (5722 exams per 1156877 Veterans; $95 \% \mathrm{CI}=4.82$ to 5.08); 8.03 scans in 2015 (9340 exams per 1162480 Veterans; $95 \% \mathrm{CI}=7.87$ to 8.20 ); 15.66 scans in 2016 (18 303 exams per 1169096 Veterans; 95\% CI = 15.43 to 15.88); 27.40 scans in 2017 (32 194 exams per 1175054 Veterans; 95\% CI $=27.10$ to 27.70); and 29.57 scans in 2018 (34 639 projected exams per 1171505 Veterans; $95 \% \mathrm{CI}=29.26$ to 29.88$)\left(P_{\text {trend }}<\right.$ .001) (Figure 2).

During the study period, there was considerable geographic variation in utilization. From 2011 to 2014, the average rates were highest at VAMCs located in Rhode Island, the Midwest, South Carolina, Florida and Puerto Rico and lowest in the southwestern and central United States (Figure 3A). From 2015 to 2018, the average rates were highest at VAMCs in the Northeast, Florida, and Puerto Rico and lowest in the southwestern and central United States (Figure 3B).

\section{Sensitivity Analyses}

When we redefined lung cancer screening exams to allow for the terms "low-dose," "LDCT," or "VCAR," a total of 92869 initial and 49142 subsequent exams were identified. This yielded similar rates to our primary analysis; 0.15 scans per 1000 eligible Veterans in 2011 (167 exams per 1133183 eligible Veterans; 95\% $\mathrm{CI}=0.1$ to 0.17 ) to 34.0 scans per 1000 eligible Veterans in 2018 (39 835 exams per 1171505 eligible Veterans; 95\% CI = 33.68 to 34.33) ( $\left.P_{\text {trend }}<.001\right)$. (Supplementary Figure 1, available online).

A sensitivity analysis evaluated the possibility of missed screenings performed in Veterans eligible for Medicare. In each year, the number of exams contained at least as many screenings for Veterans age 65-66 as for Veterans age 63-64 years (Supplementary Figure 2, available online). Age stratification did not alter the observed trend $\left(P_{\text {trend }}<.001\right.$ in Veterans aged $<65$ and $\geq 65$ ) (Supplementary Table 4 and Supplementary Figures 3 and 4, available online).

\section{Discussion}

In this national VHA evaluation of lung cancer screening utilization, we found that screening rates increased from 2011 to 2018. The screening rates increased dramatically starting in 2014, which may be because of the release of the USPSTF lung cancer screening recommendation in January 2014. The rates of screening were highest at VAMCs located in Rhode Island, the Midwest, South Carolina, Florida and Puerto Rico compared with other geographic regions in 2011 to 2014, reflecting early adopters.

Based on data from January through May, the projected annual rate of screening in 2018 was 29.6 scans (95\% CI $=29.26$ to $29.88)$ per 1000 eligible Veterans nationally in the VHA ( $P_{\text {trend }}$ $<.001)$. This is low but similar to overall US utilization rates reported in 2015 to 2016 as $2 \%-4 \%(3,4)$. In a national study of lung cancer screening utilization using screening exams registered with the American College of Radiology in 2015 to 2016 and a denominator derived from the National Health Interview Survey, screening was highest in the northeast and lowest in the west, similar to our findings (3). Previous VA data collected prospectively suggest a utilization rate of $11.6 \%$ across 2 years at select VAMCs that received dedicated resources to implement lung cancer screening (9). The rates in the present study are lower likely because they represent national, annual rates of all VAMCs.

Despite published guidelines recommending lung cancer screening for high-risk individuals, there remains a profound gap between recommended and delivered care. The reasons for low utilization in the VHA, as in other health-care systems, are likely multifactorial and exist at the patient, provider, and health-care system levels. At the patient level, awareness and recognition of lung cancer screening eligibility is key. Once patients are aware of screening, individual values and beliefs, distance to a screening facility, financial constraints, or other personal reasons may affect an individual's decision to be screened, and these factors may have contributed to the utilization rates we observed (13). In the VHA's demonstration project, 
Table 3. Facility characteristics

\begin{tabular}{|c|c|c|}
\hline Facility characteristics $^{a}$ & $\begin{array}{l}\text { Total No. of } \\
\text { facilities }\end{array}$ & $\begin{array}{l}\text { No. of facilities } \\
\text { performing } \\
\text { screenings (\%) }\end{array}$ \\
\hline Total & 130 & $75(57.7)$ \\
\hline \multicolumn{3}{|l|}{$\begin{array}{l}\text { US Census Geographic } \\
\text { Region }\end{array}$} \\
\hline Midwest & 27 & $12(44.4)$ \\
\hline VISN $10^{b}$ & 11 & $4(36.4)$ \\
\hline VISN 12 & 8 & $3(37.5)$ \\
\hline VISN 15 & 2 & $2(100.0)$ \\
\hline VISN 23 & 6 & $3(50.0)$ \\
\hline Northeast & 23 & $16(69.6)$ \\
\hline VISN 1 & 8 & $6(75.0)$ \\
\hline VISN 2 & 6 & $5(83.3)$ \\
\hline VISN 4 & 9 & $5(55.6)$ \\
\hline South & 48 & $31(64.6)$ \\
\hline VISN 5 & 6 & $3(50.0)$ \\
\hline VISN 6 & 7 & $6(85.7)$ \\
\hline VISN 7 & 8 & $5(62.5)$ \\
\hline VISN 8 & 7 & $7(100.0)$ \\
\hline VISN 9 & 5 & $3(60.0)$ \\
\hline VISN 16 & 8 & $3(37.5)$ \\
\hline VISN 17 & 7 & $4(57.1)$ \\
\hline West & 32 & $16(50.0)$ \\
\hline VISN 19 & 8 & $2(25.0)$ \\
\hline VISN 20 & 8 & $5(62.5)$ \\
\hline VISN 21 & 8 & $4(50.0)$ \\
\hline VISN 22 & 8 & $5(62.5)$ \\
\hline \multicolumn{3}{|l|}{ Complexity level $^{\mathrm{c}}$} \\
\hline $1 \mathrm{a}$ & 39 & $31(79.5)$ \\
\hline $1 b$ & 19 & $13(68.4)$ \\
\hline $1 c$ & 19 & $8(42.1)$ \\
\hline 2 & 25 & $11(44.0)$ \\
\hline 3 & 28 & $12(42.9)$ \\
\hline
\end{tabular}

aUnique facilities are provided by US region; percentages are row percentages. Example: there are 32 facilities in the western United States of which 16 (50.0\%) performed lung cancer screening, which are distributed below in the following VISNs.

bVISN = Veterans Integrated Service Network (see Supplementary Table 2, available online for VISN geographic distribution by state).

'VAMC complexity score consists of five complexity levels: 1a, 1b, 1c, 2, and 3, where $1 \mathrm{a}$ is the most complex and 3 is the least complex. This ranking system takes the following into consideration: (1) volume and patient case mix, (2) clinical services provided, (3) patient risk calculated from VA patient diagnosis, (4) total resident slots, (5) an index of multiple residency programs at a single facility, (6) total amount of research dollars, and (7) the number of specialized clinical services (10).

only $57.7 \%$ of eligible Veterans agreed to be screened and reasons for declining screening are unknown (9). At the provider level, awareness of lung cancer screening and knowledge of the guidelines is also critical (14). Studies of VHA providers found that knowledge of the USPSTF guidelines and Centers for Medicare \& Medicaid Services (CMS) coverage criteria is low (14, $15)$, and providers who lack knowledge are less likely to perform screening (14). Providers must also recognize that screening is worthwhile and feasible (13). Additionally, providers are tasked with discussing the potential benefits and risks of screening during shared decision making (currently mandated for Medicare coverage). Because many older patients who are current or former smokers also have clinically significant comorbidities, time constraints at the clinical visit is a commonly cited barrier among providers (15-24). A challenge at any point along this continuum of care could have impacted the utilization rates we observed (13).

At the health-care system level, potential barriers identified in the VHA include difficulty identifying eligible individuals in the EHR because of the relatively unstructured data capture for tobacco use, a challenge for many health-care systems $(9,25)$. The VHA's demonstration project found that $39 \%$ of patients had inaccurate or missing information on smoking status or tobacco history in the EHR (9). A lack of resources at some VAMCs may also be a barrier. The VHA's demonstration project suggested that screening at the population level would likely require multiple types of additional resources including equipment, personnel, staff training, and engagement with specialty services $(9,26)$.

The VHA has identified useful strategies to facilitate screening $(9,26)$. The VHA's demonstration project concluded that lung cancer screening coordinators are needed for populationbased lung cancer screening (9); these coordinators are part of centralized programs at each VAMC in which the clinical coordinator helps to manage screening-related activities. This allows for better coordination between all the disciplines involved in the veteran's care and reduces the number of tasks for primary care providers (27). Additionally, electronic tracking systems have the potential to organize screening results, downstream evaluations of suspicious findings, and patient follow-up (27). The VHA is currently in the process of deploying lung cancer screening tracking systems within the EHR for seamless coordination of patient care.

Perhaps the fastest measure the VHA could take to increase utilization would be to establish lung cancer screening with LDCT as a "best practice" or quality measure. Quality metrics are common for a wide range of diseases and preventive care (28-30). CMS has developed core quality measures for cervical, breast, and colorectal cancer screening (31). VHA has long used quality measures to encourage tobacco treatment, even incorporating provider incentives (32-34). A quality measure could dramatically increase VHA screening rates.

Our study is one of the first that characterizes types of ordering providers. It is not surprising that of the total number of initial screenings ordered, most were ordered by physicians in primary care/internal medicine since this specialty commonly addresses preventive care. It is also not surprising that specialists ordered a relatively small proportion of screenings because many of the patients they see have conditions that would preclude screening.

We found that 75 out of 130 unique VAMCs performed initial lung cancer screening. Notably, most of these facilities were of the highest complexity level, which may reflect availability of downstream resources needed to manage screening findings, such as access to pulmonology or thoracic surgery. Our findings may also reflect coding practices across different VAMCs; the G0297 code was not in use until 2015. Finally, given that this is a new screening, some VAMCs may not have performed this screening during the study period.

There are limitations of our study. Although we were conservative in our definition of a screening exam, misclassification of exams may have occurred. The PPV of our screening algorithm was $92 \%$. A sensitivity analysis was performed to include all LDCTs, which showed a modest increase in screening rates. We were unable to include Veterans screened in the community by non-VHA providers, but a sensitivity analysis is reassuring in that screenings did not diminish at or after age 65 years when Veterans may have been screened in the community as new Medicare beneficiaries. The estimation of our denominator is 


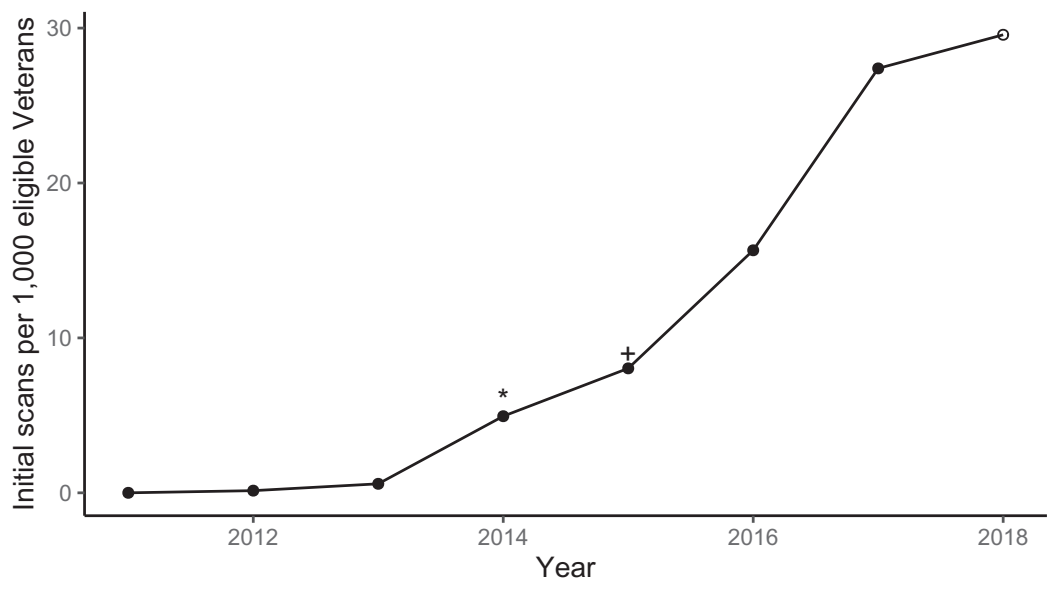

- Actual o Projected based on Jan-May

$\begin{array}{rrrrrrrrr} & 2011 & 2012 & 2013 & \mathbf{2 0 1 4} & \mathbf{2 0 1 5} & \mathbf{2 0 1 6} & \mathbf{2 0 1 7} & \mathbf{2 0 1 8} \\ \text { \# LCS exams } & 0 & 160 & 667 & 5,722 & 9,340 & 18,303 & 32,194 & 34,639 \\ \text { \# eligible Veterans } & 1,133,183 & 1,142,508 & 1,148,060 & 1,156,877 & 1,162,480 & 1,169,096 & 1,175,054 & 1,171,505\end{array}$

Figure 2. Annual low-dose computed tomography screening utilization in the Veterans Health Administration. Time period: January 1, 2011 to May 31 , 2018. The number of exams shown in the table for 2018 is projected based on the screening rate from January through May of that year. LCS = lung cancer screening; ${ }^{*}$ refers to release of the US Preventive Services Task Force Grade B recommendation for lung cancer screening with low-dose CT; + refers to release of the Centers for Medicare \& Medicaid Services Decision to cover reimbursement for lung cancer screening with low-dose CT.

\section{A $2011-2014$}

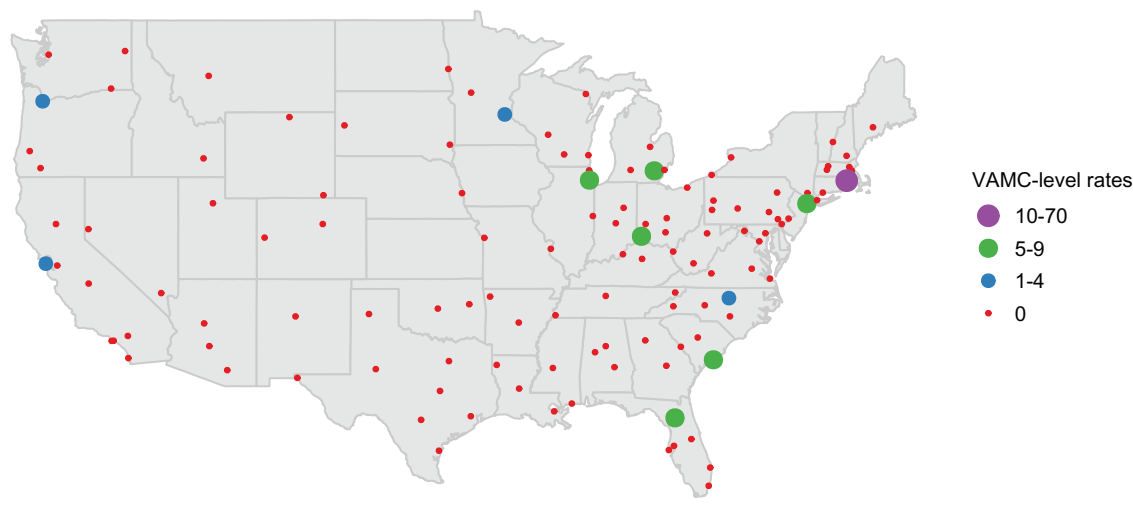

\section{B 2015-2018}

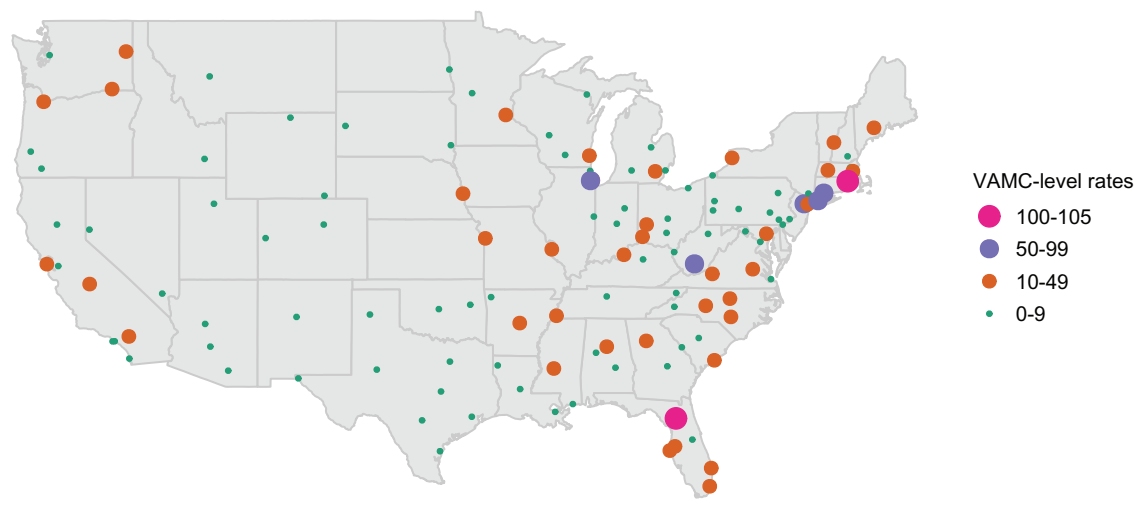

Figure 3. Geographic variation in low-dose computed tomography screening utilization in the Veterans Health Administration. Panel A provides the average annual number of initial screens per 1000 eligible Veterans in 2011 through 2014 at each VAMC. Panel B provides the same average rates in 2015 through May 2018. The map shows only the contiguous United States. The average numbers of screenings per 1000 eligible Veterans at VAMCs outside this area are as follows: Manila, Philippines are 0 in both 2011-2014 and 2015-2018; Honolulu, Hawaii are 0 in 2011-2014 and 6 in 2015-2018; Anchorage, Alaska 0 in 2011-2014 and 3 in 2015-2018; San Juan, Puerto Rico are 28 in 2011-2014 and 35 in 2015-2018. VAMC = VA medical center. 
imperfect. Documentation of smoking history in the EHR does not consistently capture pack-years and quit dates (25). Therefore, our denominator is an estimation of the percentage of Veterans who met the smoking eligibility based on a prior VHA study (9). However, this denominator is derived from actual counts of unique individuals unlike survey-estimated denominators in prior studies $(3,4)$. Data elements may be imperfect as this is administrative data. Finally, this study represents the veteran population and may not be generalizable.

Lung cancer remains the leading cause of cancer mortality in the United States, and many Veterans are at high risk for lung cancer. We found that lung cancer screening utilization within the VHA is low, but is increasing over time. Lung cancer screening with LDCT is a recommended screening for high-risk individuals and the development of a quality measure could increase utilization. Additional future interventions are needed at the patient, provider, and health-care system levels to improve lung cancer outcomes in the VHA.

\section{Funding}

This work was supported by the US Department of Veterans Affairs (VA) Office of Rural Health (ORH). Visit www.ruralhealth.va.gov to learn more. This study was also supported in part by the Vanderbilt CTSA grant UL1 TR000445 from the National Center for Advancing Translational Sciences, National Institutes of Health and the Veterans Affairs Office of Academic Affiliations.

\section{Notes}

Role of the funder: The funder had no role in the design of the study; the collection, analysis, and interpretation of the data; the writing of the manuscript; and the decision to submit the manuscript for publication. The Office of Rural Health had the opportunity to review the manuscript prior to submission for publication.

Disclosures: Dr Slatore is supported by resources from the VA Portland Health Care System in Portland, Oregon. He is the medical co-director of the lung cancer screening programs at the institution where he is employed but does not receive additional compensation for this role. All other authors have no disclosures.

Disclaimer: The contents are solely the responsibility of the authors and do not necessarily represent official views of the National Center for Advancing Translational Sciences, the National Institutes of Health, or the Veterans Health Administration.

Acknowledgments: This material is based upon work supported by the US Department of Veterans Affairs (VA) Office of Rural Health (ORH), VA Office of Academic Affiliations, VA National Quality Scholars Program, and with resources and use of facilities at VA Tennessee Valley Healthcare System in Nashville, Tennessee and VINCI.

Author contributions: JAL: conceptualization, methodology, investigation, project administration, formal analysis, validation, visualization, funding acquisition, writing - original draft, writing - review \& edit; LRS: methodology, formal analysis, validation, visualization, writing - review \& editing; JD: software, data curation, validation; GCE: formal analysis, validation, writing review \& editing; MEM: methodology, data curation, resources, writing - review \& editing; AM: conceptualization, resources, writing - review \& editing; CGS: methodology, writing - review \& editing; EG: resources, writing - review \& editing; JK: methodology, writing - review \& editing; RHS: conceptualization, methodology, resources, writing - review \& editing; RSD: resources, writing - review \& editing, funding acquisition; PPM: methodology, validation, writing - review \& editing; LK: conceptualization, methodology, writing - review \& editing; SN: conceptualization, methodology, writing - review \& editing; CLR: conceptualization, methodology, investigation, validation, supervision, writing - original draft, writing - review \& edit.

\section{Data availability}

The data underlying this article are available in the article and in its online supplementary material.

\section{References}

1. American Cancer Society. Cancer Facts \& Figures 20192019 ed. Atlanta, GA: American Cancer Society; 2019.

2. National Lung Screening Trial Research Team, Aberle DR, Adams AM, Berg $\mathrm{CD}$, et al Reduced lung-cancer mortality with low-dose computed tomographic screening. N Engl J Med. 2011;365(5):395-409.

3. Pham D, Bhandari S, Oechsli M, Pinkston CM, Kloecker GH. Lung cancer screening rates: data from the Lung Cancer Screening Registry. J Clin Oncol. 2018;36(suppl 15):6504-6504.

4. Jemal A, Fedewa SA. Lung cancer screening with low-dose computed tomography in the United States-2010 to 2015. JAMA Oncol. 2017;3(9):1278-1281.

5. Brown DW. Smoking prevalence among US veterans. J Gen Intern Med. 2010; 25(2):147-149.

6. Odani S, Agaku IT, Graffunder CM, Tynan MA, Armour BS. Tobacco product use among military veterans-United States, 2010-2015. MMWR Morb Mortal Wkly Rep. 2018;67(1):7-12.

7. Moyer VA, Force U. Screening for lung cancer: U.S. Preventive Services Task Force recommendation statement. Ann Intern Med. 2014;160(5):330-338.

8. Kinsinger LS, Atkins D, Provenzale D, Anderson C, Petzel R. Implementation of a new screening recommendation in health care: the Veterans Health Administration's approach to lung cancer screening. Ann Intern Med. 2014; 161(8):597-598.

9. Kinsinger LS, Anderson C, Kim J, et al Implementation of lung cancer screening in the Veterans Health Administration. JAMA Intern Med. 2017;177(3): 399-406.

10. Veterans Health Administration. Summary of VHA Facility Complexity Model. 2014. https://www.vendorportal.ecms.va.gov/FBODocumentServer/ DocumentServer.aspx? DocumentId=2793591\&FileName=VA118-16-R-1059A00002002.docx Accessed July 9, 2019.

11. Wilson EB. Probable inference, the law of succession, and statistical inference. J Am Stat Assoc. 1927;22(158):209-212.

12. Brown LD, Cai TT, DasGupta A. Interval estimation for a binomial proportion. Stat Sci. 2001;16(2):101-133.

13. Cabana MD, Rand CS, Powe NR, et al Why don't physicians follow clinical practice guidelines? A framework for improvement. JAMA. 1999;282(15): 1458-1465.

14. Lewis JA, Chen H, Weaver KE, et al Low provider knowledge is associated with less evidence-based lung cancer screening. J Natl Compr Canc Netw. 2019; 17(4):339-346.

15. Triplette M, Kross EK, Mann BA, et al An assessment of primary care and pulmonary provider perspectives on lung cancer screening. Annals Ats. 2018; 15(1):69-75.

16. Lewis JA, Petty WJ, Tooze JA, et al Low-dose CT lung cancer screening practices and attitudes among primary care providers at an academic medical center. Cancer Epidemiol Biomarkers Prev. 2015;24(4):664-670.

17. Duong DK, Shariff-Marco S, Cheng I, et al Patient and primary care provider attitudes and adherence towards lung cancer screening at an academic medical center. Prev Med Rep. 2017;6(2017):17-22.

18. Eberth JM, McDonnell KK, Sercy E, et al A national survey of primary care physicians: perceptions and practices of low-dose CT lung cancer screening. Preu Med Rep. 2018;11(2018):93-99.

19. Kanodra NM, Pope C, Halbert CH, Silvestri GA, Rice LJ, Tanner NT. Primary care provider and patient perspectives on lung cancer screening. A qualitative study. Ann Am Thorac Soc. 2016;13(11):1977-1982.

20. Rajupet S, Doshi D, Wisnivesky JP, Lin JJ. Attitudes about lung cancer screening: primary care providers versus specialists. Clin Lung Cancer. 2017;18(6): e417-e423.

21. Henderson LM, Jones LM, Marsh MW, et al Opinions, practice patterns, and perceived barriers to lung cancer screening among attending and resident primary care physicians. Risk Manag Healthc Policy. 2018;10:189-195. 
22. Zeliadt SB, Hoffman RM, Birkby G, et al Challenges implementing lung cancer screening in federally qualified health centers. Am J Prev Med. 2018;54(4):568-575.

23. Hoffman RM, Sussman AL, Getrich CM, et al Attitudes and beliefs of primary care providers in New Mexico about lung cancer screening using low-dose computed tomography. Prev Chronic Dis. 2015;12(E108):1-11.

24. Khairy M, Duong DK, Shariff-Marco S, et al An analysis of lung cancer screening beliefs and practice patterns for community providers compared to academic providers. Cancer Control. 2018;25(1):107327481880690.

25. Modin HE, Fathi JT, Gilbert CR, et al Pack-year cigarette smoking history for determination of lung cancer screening eligibility. Comparison of the electronic medical record versus a shared decision-making conversation. Ann Am Thorac Soc. 2017;14(8):1320-1325.

26. Jackson GL, King HA, McNeil RB, et al Evaluation of the VA Lung Cancer Screening Clinical Demonstration Project - Report Submitted to the Veterans Affairs Office of the under Secretary for Health. Durham, NC: Veterans Affairs Center for Health Services Research in Primary Care and National Center for Health Promotion and Disease Prevention; 2016.

27. McKee BJ, McKee AB, Kitts AB, Regis SM, Wald C. Low-dose computed tomography screening for lung cancer in a clinical setting: essential elements of a screening program. J Thorac Imaging. 2015;30(2):115-129.
28. Bravata DM, Myers LJ, Arling G, et al Quality of care for veterans with transient ischemic attack and minor stroke. JAMA Neurol. 2018;75(4): 419-427.

29. Rosenberg RD, Yankaskas BC, Abraham LA, et al Performance benchmarks for screening mammography. Radiology. 2006;241(1):55-66.

30. Fayad NF, Kahi CJ. Quality measures for colonoscopy: a critical evaluation. Clin Gastroenterol Hepatol. 2014;12(12):1973-1980.

31. Centers for Medicare and Medicaid Services. ACO and PCMH/Primary Care Measures. 2016; Version 1.0. https://www.cms.gov/Medicare/QualityInitiatives-Patient-Assessment-Instruments/QualityMeasures/Downloads/ ACO-and-PCMH-Primary-Care-Measures.pdf. Accessed August 5, 2019.

32. Sherman SE. A framework for tobacco control: lessons learnt from Veterans Health Administration. BMJ. 2008;336(7651):1016-1019.

33. Smith MW, Chen S, Siroka AM, Hamlett-Berry K. Using policy to increase prescribing of smoking cessation medications in the VA healthcare system. Tob Control. 2010;19(6):507-511.

34. Hamlett-Berry K, Davison J, Kivlahan DR, Matthews MH, Hendrickson JE, Almenoff PL. Evidence-based national initiatives to address tobacco use as a public health priority in the Veterans Health Administration. Mil Med. 2009; 174(1):29-34. 